\title{
On the Method of Determining Onboard Ephemeris Data L1OC and L3OC GLONASS Aimed at Testing Positioning Algorithms in the GNSS Receiver
}

\author{
Taliya G. Sharfunova and Daria A. Krasilnikova* \\ Joint Stock Company «Construction Bureau \\ of Navigation Systems» \\ Moscow, Russian Federation
}

Received 23.05.2019, received in revised form 28.10.2019, accepted 21.01.2020

Abstract. The paper considers a method of determining GLONASS ephemeris data in the L1OC and L3OC digital form aimed at testing the algorithms of accurate navigation determinations in consumer navigation equipment. The task of determining long-term motion model parameters of a navigation spacecraft is set as nonlinear problem of designing a matching model. This task is unstable and according to the analysis is categorized as incorrect. The application of a traditional least squares method to determine the long-term motion model parameters of a navigation spacecraft does not allow to obtain equally accurate solutions of condition equations system when initial conditions and/ or iterations amount have been changed. In this respect, Tikhonov's regularization method has been carried out. It is based on the application of additional prior information. The obtained results have been tested by the comparison of estimated navigation spacecraft position according to the adjusted (speed and acceleration) ephemeris data, long-term motion model parameters and SP3 final reference ephemeris published on the SVOEVP website. The long-term motion model parameters of GLONASS navigation spacecraft that were defined by regularizing algorithms, have allowed to calculate the position of navigation spacecraft orbital grouping in terms of four-hours fitting intervals within $0,2 \mathrm{~m}$ tolerance (maximum deviations according to module of estimated navigation spacecraft position from SVOEVP SP3 reference ephemeris).

Keywords: GLONASS ephemeris data, L1OC and L3OC navigation data, long-term motion model parameters, GNSS receiver, SVOEVP, matching model, regularizing algorithms.

Citation: Sharfunova T.G., Krasilnikova D.A. On the method of determining onboard ephemeris data L1OC and L3OC GLONASS aimed at testing positioning algorithms in the GNSS receiver, J. Sib. Fed. Univ. Eng. \& Technol., 2020, 13(7), 788-802. DOI: 10.17516/1999-494X-0228

(c) Siberian Federal University. All rights reserved

This work is licensed under a Creative Commons Attribution-Non Commercial 4.0 International License (CC BY-NC 4.0).

* Corresponding author E-mail address: 9135500382@mail.ru 


\title{
О способе определения бортовой
}

эфемеридной информации L1OC, L3OC ГЛOНАCC

\section{в целях тестирования алгоритмов местоопределения \\ в навигационном приемнике ГЛОНАСС}

\author{
Т.Г. Шарфунова, Д.А. Красильникова \\ Акционерное общество \\ «Конструкторское бюро навигачионных систем» \\ Российская Федерация, Москва
}

\begin{abstract}
Аннотация. В работе рассматривается способ определения эфемеридной информации (ЭИ) HKA в формате цифровой информации L1OC, L3OC ГЛОНАCC для применения в целях тестирования алгоритмов точных навигационных определений в НАП. Задача определения параметров долговременной модели движения (ПДМД) НКА ГЛОНАСС сформулирована как нелинейная задача построения согласующей модели. Указанная задача является неустойчивой и, как показал анализ, относится к классу некорректных задач. Использование классического метода наименьших квадратов (МНК) для определения ПДМД НКА не позволяет получить эквивалентные по точности решения системы условных уравнений при изменении начальных условий и/или количества итераций. В связи с этим для определения ПДМДНКА был применен метод регуляризации по А.Н. Тихонову, основанный на применении дополнительной априорной информации. Полученные результаты протестированы сравнением расчетного положения НКА по уточненной (в части скоростей и ускорений) ЭИ, ПДМД и окончательных опорных эфемерид SP3, публикуемых на сайте СВОЭВП. ПДМД НКА ГЛОНАСС, определенные с применением регуляризирующих алгоритмов, позволили обеспечить расчет положения НКА орбитальной группировки (ОГ) ГЛОНАСС на интервалах согласования до 4 ч с погрешностью, не превышающей 0,2 м (максимальные по модулю отклонения расчетного положения НКА от опорных эфемерид SР3 СВОЭВП).
\end{abstract}

Ключевые слова: эфемеридная информация ГЛОНАСС, формат навигационного сообщения L1OC и L3OC, параметры долговременной модели движения, навигационный приемник, СВОЭВП, согласующая модель, регуляризирующие алгоритмы.

Цитирование: Шарфунова, Т.Г. О способе определения бортовой эфемеридной информации L1OC, L3OC ГЛОНАCC в целях тестирования алгоритмов местоопределения в навигационном приемнике ГЛОНАСС / Т.Г. Шарфунова, Д.А. Красильникова // Журн. Сиб. федер. ун-та. Техника и технологии, 2020. 13(7). С. 788-802. DOI: 10.17516/1999494X-0228

\section{Введение}

Возможности навигации потребителей зависят от точности эфемерид в составе навигационного сообщения (НС), транслируемого с борта НКА. В 2020 г. планируется запуск НКА «Глонасс-К2» с повышенными тактико-техническими характеристиками и проведение летноконструкторских испытаний. В составе НС перспективных сигналов с кодовым разделением в частотных диапазонах L1 и L3 ГЛОНАСС с борта НКА «Глонасс-К2» предусмотрена передача согласованной (в части скоростей и ускорений) оперативной ЭИ с повышенной разрядностью представления.

До начала реального излучения новых сигналов с борта НКА «Глонасс-К2» алгоритмы использования ЭИ в формате HC L1OC, L3OC должны быть отработаны в навигационных прием-

$$
-789-
$$


никах. Если не требуется отработка точности навигационных определений по HC L1OC, L3OC, то для генерации НС могут быть использованы опорные эфемериды НКА ГЛОНАСС, предоставляемые в апостериорном режиме Центрами обработки СВОЭВП и ЦНИИМаш в формате SP3 файлов. Указанные эфемериды рассчитываются по сложным моделям движения с учетом большого числа возмущающих воздействий на движение НКА, обеспечивают наивысшую точность расчета положения НКА на опорные эпохи, но не обеспечивают высокие точности расчета положения НКА по алгоритмам потребителя, так как в навигационных приемниках для расчета положения НКА используют более простые модели.

В составе навигационной оперативной информации L1OC, L3OC предусмотрена передача согласованных эфемерид НКА, которые на те же моменты времени $t_{b}$ должны быть рассчитаны на основе опорных эфемерид таким образом, чтобы методические ошибки прогнозирования по более простым моделям движения НКА, используемым потребителем, в среднем на интервалах до 15 мин от опорной эпохи $t_{b}$ были минимальны.

Кроме этого, в составе неоперативной информации НКА «Глонасс-К2» планируется передача ПДМД НКА, которые должны обеспечить точность расчета положения НКА по упрощенным алгоритмам потребителя не более 1 м на интервалах расчета положения НКА до 4 ч.

Целью работы является совершенствование определения ПДМД НКА для целей моделирования навигационной информации по перспективным сигналам L1OC, L3OC системы ГЛОHACC.

Задача определения ПДМД НКА ГЛОНАСС сформулирована как нелинейная задача построения согласующей модели на мерном интервале 4 ч. Использование классического МНК для определения ПДМД не позволяет получить эквивалентные по точности решения системы условных уравнений при изменении начальных условий и/или количества итераций. Как показал анализ, указанная задача неустойчива и относится к классу некорректных задач. В связи с этим для определения ПДМД был применен метод регуляризации по А.Н. Тихонову, основанный на применении дополнительной априорной информации. Результаты решений протестированы сравнением расчетного положения НКА ОГ ГЛОНАСС по уточненной (в части скоростей и ускорений) ЭИ, ПДМД и окончательных опорных эфемерид SP3 CВОЭВП. Примененный способ позволил получить устойчивые решения для 12 уточняемых параметров долговременной модели движения НКА. Максимальная погрешность расчета положения НКА ОГ ГЛОНАСС на интервалах до +4 ч от опорной эпохи $t_{b}$ не превысила 0,2 м по более чем 5700 реализациям уточнения ПДМД.

\section{Определение согласованных эфемерид и ПДМД НКА ГЛОНАСС для генерации навигационного сообщения L1OC, L3OC}

При определении местоположения в навигационном приемнике текущее положение НКА ГЛОНАСС рассчитывается по оперативной ЭИ из НС методом Рунге-Кутта по уравнениям движения НКА, которые приведены в [1].

Согласованная эфемеридная информация и ПДМД НКА ГЛОНАСС, передаваемые в составе HC L1OC и L3OC, предназначены для расчета положения HKA на интервалах до 4 ч и включают в себя параметры вида $x, y, z, \dot{x}, \dot{y}, \dot{z}, \ddot{x}, \ddot{y}, \ddot{z}$ и $a_{X 0}, a_{X 1}, a_{X 2}, a_{X 3}, a_{X 4}, a_{Y 0}, a_{Y 1}, a_{Y 2}, a_{Y 3}, a_{Y 4}$, $a_{Z 0}, a_{Z 1}, a_{Z 2}, a_{Z 3}, a_{Z 4}$, заданные на опорное время $t_{b}$. 
Здесь $x, y, z, \dot{x}, \dot{y}, \dot{z}$ - составляющие векторов координат и скорости центра масс НКА ГЛОНАСС на опорную эпоху $t_{b}$ в СК ПЗ-90.11, $\ddot{x}, \ddot{y}, \ddot{z}$ - составляющие вектора ускорения НКА в СК П3-90.11, далее следуют 12 параметров долговременной модели движения.

Дополнительные ускорения НКА $a_{X}\left(t, t_{b}\right), a_{Y}\left(t, t_{b}\right), a_{Z}\left(t, t_{b}\right)$ на текущий момент времени $t$ рассчитываются потребителем с использованием ПДМД, которые являются коэффициентами полиномов 4-й степени для разницы между текущим временем $t$ и опорным временем $t_{b}$, по алгоритмам, приведенным в [1]:

$$
\begin{aligned}
& a_{X}\left(t, t_{b}\right)=a_{X 0}+a_{X 1} \cdot\left(t-t_{b}\right)+a_{X 2} \cdot\left(t-t_{b}\right)^{2}+a_{X 3} \cdot\left(t-t_{b}\right)^{3}+a_{X 4} \cdot\left(t-t_{b}\right)^{4}, \\
& a_{Y}\left(t, t_{b}\right)=a_{Y 0}+a_{Y 1} \cdot\left(t-t_{b}\right)+a_{Y 2} \cdot\left(t-t_{b}\right)^{2}+a_{Y 3} \cdot\left(t-t_{b}\right)^{3}+a_{Y 4} \cdot\left(t-t_{b}\right)^{4}, \\
& a_{Z}\left(t, t_{b}\right)=a_{Z 0}+a_{Z 1} \cdot\left(t-t_{b}\right)+a_{Z 2} \cdot\left(t-t_{b}\right)^{2}+a_{Z 3} \cdot\left(t-t_{b}\right)^{3}+a_{Z 4} \cdot\left(t-t_{b}\right)^{4} .
\end{aligned}
$$

Задача построения согласованных эфемерид на опорные эпохи $t_{b}$ сводится к итерационному решению линеаризованной задачи:

$$
\vec{u}=\arg \min _{u}\left(\|A \vec{u}-\vec{f}\|_{2}^{2}\right) \vec{u} .
$$

Здесь $A$ - вещественная матрица размерности $m \times n$ (матрица частных производных), $\vec{u}$ и $\vec{f}$ - векторы размерности $n$ и $m$ соответственно, $\vec{u}$ - уточняемый вектор состояния НКА, $\vec{f}-$ вектор правых частей (вектор невязок). Задача решается в условиях, когда входные данные $\{A$, $\vec{f}\}$ возмущены.

Уточняемый вектор $\vec{u}$ включает в себя составляющие векторов скорости и ускорений НКА $\{\dot{x}, \dot{y}, \dot{z}, \ddot{x}, \ddot{y}, \ddot{z}\}$, заданные в СК ПЗ-90.11 на опорную эпоху $t_{b}$ и ПДМД НКА ГЛОНАСС в составе $a_{X 0}, a_{X 1}, a_{X 2}, a_{X 3}, a_{X 4}, a_{Y 0}, a_{Y 1}, a_{Y 2}, a_{Y 3}, a_{Y 4}, a_{Z 0}, a_{Z 1}, a_{Z 2}, a_{Z 3}, a_{Z 4}$. Параметры $a_{X 0}, a_{Y 0}, a_{Z 0}$, передаваемые с борта НКА, предназначены для компенсации погрешности представления компонент ускорения $\ddot{x}, \ddot{y}, \ddot{z}$ из-за усечения разрядности в $\mathrm{HC}$ L1OC, L3OC, и поэтому параметры $a_{X 0}, a_{Y 0}$, $a_{Z 0}$ не требуется включать в состав уточняемого вектора.

Уточнение вектора $\vec{u}$ проводилось в два этапа. На 1-м этапе на интервале согласования 0,5 ч $\left[t_{b}-0,25 u ; t_{b}+0,25\right.$ u с использованием линеаризованной математической модели $A \vec{u}_{1}=\vec{f}_{1}$ были получены оценки вектора состояния НКА $\vec{u}_{1}$ в составе $\{\dot{x}, \dot{y}, \dot{z}, \ddot{x}, \ddot{y}, \ddot{z}\}$ (согласованные скорости и ускорения).

Так как излучаемые в составе реального навигационного сообщения эфемериды недостаточно точны, в качестве опорной орбиты НКА были взяты точные эфемериды $x\left(t_{b}\right), y\left(t_{b}\right), z\left(t_{b}\right)$, в расчетах использовали окончательные данные опорных эфемерид НКА с сайта СВОЭВП [2] на фиксированные опорные эпохи $t_{b}$. Положение НКА на текущий момент времени $t$ на интервале согласования рассчитывали при помощи интерполяционного многочлена Лагранжа 12-го порядка [3].

В общем случае вместо указанных данных могут быть использованы эфемериды НКА, спрогнозированные в инерциальной системе координат на требуемые моменты времени в полном поле сил, где ускорение НКА описывают вектором дифференциального уравнения

$$
\begin{aligned}
\vec{r}_{\text {sat }}=\vec{a}_{\text {Earth }}+\vec{a}_{\text {Sun }}+\vec{a}_{\text {Moon }} & +\vec{a}_{S R P}+\vec{a}_{\text {tides }}+\vec{a}_{N G P} \vec{a}_{\text {Earth }}, \\
& -791-
\end{aligned}
$$


где $\vec{a}_{E a r t h}-$ ускорения из-за несферичности Земли, рассчитываются по разложениям в ряд по сферическим функциям не ниже восьмого порядка и степени включительно; $\vec{a}_{\text {Sun }}, \vec{a}_{\text {Moоn }}-$ ускорения от солнечных и лунных гравитационных возмущений; $\vec{a}_{S R P}$ - ускорения от сил радиационного давления на корпус НКА (прямого и отраженного излучения Солнца); $\vec{a}_{\text {tides }}-$ ускорения из-за влияния лунно-солнечных приливных вариаций гравитационного поля Земли; $\vec{a}_{N G P}-$ ускорения НКА от сил негравитационной природы, определяемые эмпирически.

В качестве начального априорного значения уточняемого вектора состояния НКА $\vec{u}_{1}$ при расчетах на первой итерации были приняты составляющие вектора скорости и ускорения НКА в системе координат (СК) ПЗ-90.11 на опорную эпоху $t_{b}: \dot{x}\left(t_{b}\right), \dot{y}\left(t_{b}\right), \dot{z}\left(t_{b}\right)$, рассчитанные при помощи интерполяционного многочлена Лагранжа [3] по значениям опорных эфемерид НКА ГЛОНАСС SP3 СВОЭВП $x\left(t_{b}\right), y\left(t_{b}\right), z\left(t_{b}\right)$, и составляющие вектора ускорений НКА $\ddot{x}\left(t_{b}\right), \ddot{y}\left(t_{b}\right), \ddot{z}\left(t_{b}\right)$ от солнечных и лунных гравитационных возмущений, рассчитанные по алгоритмам, приведенным в [1] (прил. К, У).

Матрица частных производных $A$ при уточнении вектора $\vec{u}_{1}$ рассчитана методом конечных приращений, $k$ - количество эпох на получасовом интервале, для которых рассчитаны частные производные:

$$
A=\left[\begin{array}{cccccc}
\frac{\left(\delta x_{1}\right)}{2(\delta \dot{x})} & \frac{\left(\delta x_{1}\right)}{2(\delta \dot{y})} & \frac{\left(\delta x_{1}\right)}{2(\delta \dot{z})} & \frac{\left(\delta x_{1}\right)}{2(\delta \ddot{x})} & \frac{\left(\delta x_{1}\right)}{2(\delta \ddot{y})} & \frac{\left(\delta x_{1}\right)}{2(\delta \ddot{z})} \\
\frac{\left(\delta y_{1}\right)}{2(\delta \dot{x})} & \frac{\left(\delta y_{1}\right)}{2(\delta \dot{y})} & \frac{\left(\delta y_{1}\right)}{2(\delta \dot{z})} & \frac{\left(\delta y_{1}\right)}{2(\delta \ddot{x})} & \frac{\left(\delta y_{1}\right)}{2(\delta \ddot{y})} & \frac{\left(\delta y_{1}\right)}{2(\delta \ddot{z})} \\
\ldots & \ldots & \ldots & \ldots & \ldots & \ldots \\
\frac{\left(\delta z_{3 k}\right)}{2(\delta \dot{x})} & \frac{\left(\delta z_{3 k}\right)}{2(\delta \dot{y})} & \frac{\left(\delta z_{3 k}\right)}{2(\delta \dot{z})} & \frac{\left(\delta z_{3 k}\right)}{2(\delta \ddot{x})} & \frac{\left(\delta z_{3 k}\right)}{2(\delta \ddot{y})} & \frac{\left(\delta z_{3 k}\right)}{2(\delta \ddot{z})}
\end{array}\right]
$$

При расчете частных производных приращения начальных условий скоростей и ускорений в программно-математической модели использовали приращения: $|\delta \dot{x}|,|\delta \dot{y}|,|\delta \dot{z}| \sim 10^{-7} \kappa M / c$, $|\delta \ddot{x}|,|\delta \ddot{y}|,|\delta \ddot{z}| \sim 10^{-6}$ км/c. Каждые три элемента столбца матрицы $A$ на моменты времени $t_{i}$ (например, $\left.\frac{\left(\delta x_{i}\right)}{2(\delta \dot{y})}, \frac{\left(\delta y_{i}\right)}{2(\delta \dot{y})}, \frac{\left(\delta z_{i}\right)}{2(\delta \dot{y})}\right)$ определяют по результатам интегрирования уравнений движения НКА на моменты времени $t_{i}$ по алгоритму расчета К.2.1 [1] при возмущении начальных условий соответствующего уточняемого элемента с положительным и отрицательным приращением (в данном случае при значениях $\delta \dot{y}\left(t_{b}\right)=+10^{-7} \kappa M / c$ и $\left.\delta \dot{y}\left(t_{b}\right)=-10^{-7} \kappa M / c\right)$.

Систему условных уравнений $A \vec{u}_{1}=\vec{f}_{1}$ на каждой итерации решали с применением классического МНК. Для повышения численной устойчивости решения задачи предварительно было проведено масштабирование столбцов матрицы $A$ с тем, чтобы все элементы матрицы частных производных $A$ находились в диапазоне: $\left|a_{i j}\right| \leq 1$.

Чтобы процедура масштабирования не вносила дополнительные погрешности в исходные данные $\left\{A, \vec{f}_{1}\right\}$, масштабирующий множитель целесообразно брать равным $10^{-n}$, где для каждого столбца матрицы $A$ параметр $n \in \mathbb{N}$ определяется из условия $10^{n} \sim \max \left|a_{i j}\right|$. Оценка числа обусловленности cond $(A)=\|A\| \cdot\left\|A^{-1}\right\|$, проведенная с использованием сингулярного разложения по алгоритмам $[4,5]$, показала, что предлагаемая процедура масштабирования позволяет понизить число обусловленности матрицы условных уравнений $A$ с 360 до 1,3 и повысить чис- 
ленную устойчивость данной задачи, а также снизить погрешности определения оцениваемого вектора при нахождении решения системы уравнений.

Для решения системы уравнений $A^{T} A \vec{u}_{1}=A^{T} \vec{f}_{1}$ использовали алгоритм экономного пересчета треугольных сомножителей метода Холецкого (метод квадратного корня), приведенный в $[5,6]$. Сходимость итерационной последовательности оценки вектора состояния НКА $\left\{\dot{x}\left(t_{b}\right), \dot{y}\left(t_{b}\right), \dot{z}\left(t_{b}\right), \ddot{x}\left(t_{b}\right), \ddot{y}\left(t_{b}\right), \ddot{z}\left(t_{b}\right)\right\}$ зависит от начального априорного значения вектора, однако систему уравнений требуется решать не более двух-трех раз благодаря быстрой сходимости метода Ньютона. Критерием сходимости являлись значения поправок к уточняемому вектоpy $\left\{\dot{x}\left(t_{b}\right), \dot{y}\left(t_{b}\right), \dot{z}\left(t_{b}\right), \ddot{x}\left(t_{b}\right), \ddot{y}\left(t_{b}\right), \ddot{z}\left(t_{b}\right)\right\}$ на текущей итерации, не превышающие по абсолютному значению цену младшего разряда их представления в навигационной цифровой информации, а именно величин $\pm 0,001 \mathrm{M \mu} / \mathrm{c}$ и $\pm 2 \cdot 10^{-6} \mathrm{MM} / \mathrm{c}^{2}$ по компонентам скорости и ускорений НКА соответственно, а критерием успешного решения задачи считали эквивалентные по точности решения, обеспечивающие расчет положения НКА со среднеквадратическим отклонением (СКО) от опорных эфемерид СВОЭВП на 15-минутном интервале на уровне, втрое меньшем, чем уровень погрешности, заданный в Таблице К.1 [1] для упрощенного алгоритма, т.е. не более $1,6 \mathrm{~cm}$.

По согласованной (в части скоростей и ускорений) ЭИ НКА проведен расчет положения НКА на интервале согласования 0,5 ч $\left[t_{b}-0,254 ; t_{b}+0,254\right]$ и апостериорная оценка погрешности расчета положения НКА относительно опорных эфемерид SР3 СВОЭВП. Результаты оценок приведены ниже.

На 2-м этапе на интервале согласования 4 часа $\left[t_{b} ; t_{b}+4\right.$ u] был уточнен вектор $\vec{u}_{2}$ в составе $\left\{a_{X 1}, a_{X 2}, a_{X 3}, a_{X 4}, a_{Y 1}, a_{Y 2}, a_{Y 3}, a_{Y 4}, a_{Z 1}, a_{Z 2}, a_{Z 3}, a_{Z 4}\right\}$. В качестве начальных условий использовали окончательные данные опорных эфемерид НКА $x\left(t_{b}\right), y\left(t_{b}\right), z\left(t_{b}\right)$ с сайта СВОЭВП на фиксированные опорные эпохи $t_{b}$ и согласованные скорости и ускорения на опорные эпохи $t_{b}$, полученные на первом этапе.

В качестве начального априорного значения уточняемого вектора $\vec{u}_{2}$ могут быть приняты нулевые значения параметров $\left\{a_{X 1}, a_{X 2}, a_{X 3}, a_{X 4}, a_{Y 1}, a_{Y 2}, a_{Y 3}, a_{Y 4}, a_{Z 1}, a_{Z 2}, a_{Z 3}, a_{Z 4}\right\}$ или любые значения из диапазона приращений начальных условий, используемых при расчете матрицы частных производных.

Матрица частных производных $A$ при уточнении вектора $\vec{u}_{2}$ рассчитана методом конечных приращений. При расчете частных производных приращения начальных условий для вектора $\left\{a_{X 1}, a_{X 2}, a_{X 3}, a_{X 4}, a_{Y 1}, a_{Y 2}, a_{Y 3}, a_{Y 4}, a_{Z 1}, a_{Z 2}, a_{Z 3}, a_{Z 4}\right\}$ в программно-математической модели использовали значения приращений соответственно для $\left|\delta a_{X 1}\right|,\left|\delta a_{Y 1}\right|,\left|\delta a_{Z 1}\right| \sim 5 \cdot 10^{-14} \mathrm{\kappa m} / \mathrm{c}^{3}$, для $\left|\delta a_{X 2}\right|,\left|\delta a_{Y 2}\right|,\left|\delta a_{Z 2}\right| \sim 5 \cdot 10^{-18} \kappa м / c^{4}$, для $\left|\delta a_{X 3}\right|,\left|\delta a_{Y 3}\right|,\left|\delta a_{Z 3}\right| \sim 5 \cdot 10^{-22} \kappa м / c^{5}$, для $\left|\delta a_{X 4}\right|,\left|\delta a_{Y 4}\right|, \mid \delta a_{Z 4}$ $\sim 5 \cdot 10^{-27} \mathrm{kM} / \mathrm{c}^{6}$. Приращения могут быть выбраны иными, однако они должны выбираться из диапазона устойчивой области зависимости производных от приращений на одной тестовой эпохе при интегрировании на +4 ч. Здесь $k$ - количество эпох на 4-часовом интервале, для которых рассчитывают частные производные.

Анализ показал, что масштабирование матрицы $A$ позволяет значительно понизить число обусловленности - с $6 \cdot 10^{13}$ до $2,5 \cdot 10^{3}$. Однако для данного состава уточняемых параметров, несмотря на понижение числа обусловленности, матрица системы уравнений все еще является плохо обусловленной и относительная погрешность решения может возрасти примерно в 
2500 раз по сравнению с погрешностью расчета правой части при решении системы уравнений $A \vec{u}=\vec{f}$.

По смыслу постановки задачи все допустимые решения задачи $A \vec{u}=\vec{f}$ должны являться эквивалентными по точности решениями этой системы уравнений. Тестовые расчеты показали, что при использовании классического МНК для оценки вектора $\vec{u}$ в составе $\left\{a_{X 1}, a_{X 2}, a_{X 3}\right.$, $\left.a_{X 4}, a_{Y 1}, a_{Y 2}, a_{Y 3}, a_{Y 4}, a_{Z 1}, a_{Z 2}, a_{Z 3}, a_{Z 4}\right\}$ решения от итерации к итерации могут существенно различаться и зависят от задания начальных априорных значений уточняемого вектора $\vec{u}$.

Согласно $[7,8]$, такие задачи относятся к классу некорректных. В условиях, когда входные данные $\{A, f\}$ возмущены, матрица $A$ является плохо обусловленной, для отыскания устойчи-

вых приближений к элементу $u_{f}, u_{f}=\arg \min \|u\|_{B}$ широко применяется метод регуляризации $[7,8]$. При этом $B=B^{T} \geq 0, A^{T} A+B>0,\|u\|_{B}=(B u, u)^{1 / 2}$.

Регуляризованное решение системы уравнений $A \vec{u}=\vec{f}$ находится как

$$
u^{\alpha}=\arg \min _{u}\left(\|A u-f\|_{2}^{2}+\alpha \cdot\|u\|_{B}^{2}\right) .
$$

Элемент $u^{a}$ определяется из уравнения

$$
\left(A^{T} A+\alpha B\right) u=A^{T} f
$$

где $a>0$ - параметр регуляризации.

При оценке вектора $\left\{a_{X 1}, a_{X 2}, a_{X 3}, a_{X 4}, a_{Y 1}, a_{Y 2}, a_{Y 3}, a_{Y 4}, a_{Z 1}, a_{Z 2}, a_{Z 3}, a_{Z 4}\right\}$ был применен эвристический (прагматический) выбор параметра регуляризации с уменьшением значения параметра регуляризации от итерации к итерации по принципу невязки.

Априорная $B$ матрица имеет смысл ковариационной матрицы случайных ошибок уточняемого вектора $\left\{a_{X 1}, a_{X 2}, a_{X 3}, a_{X 4}, a_{Y 1}, a_{Y 2}, a_{Y 3}, a_{Y 4}, a_{Z 1}, a_{Z 2}, a_{Z 3}, a_{Z 4}\right\}$ и в начале каждой итерации полагалась диагональной.

Для повышения численной устойчивости процесса при решении уравнений $\left(A^{T} A+\alpha B\right) u=A^{T} f$ был применен метод Холецкого.

Сходимость итерационной последовательности оценки вектора состояния НКА $\left\{a_{X 1}\right.$, $\left.a_{X 2}, a_{X 3}, a_{X 4}, a_{Y 1}, a_{Y 2}, a_{Y 3}, a_{Y 4}, a_{Z 1}, a_{Z 2}, a_{Z 3}, a_{Z 4}\right\}$ зависит от начального априорного значения вектора, однако систему уравнений требуется решать не более трех-четырех раз. Критерием сходимости являлись значения поправок к уточняемому вектору $\left\{a_{X 1}, a_{X 2}, a_{X 3}, a_{X 4}, a_{Y 1}, a_{Y 2}\right.$, $\left.a_{Y 3}, a_{Y 4}, a_{Z 1}, a_{Z 2}, a_{Z 3}, a_{Z 4}\right\}$ на текущей итерации, не превышающие по абсолютному значению цену младшего разряда их представления в навигационной цифровой информации, а имен-

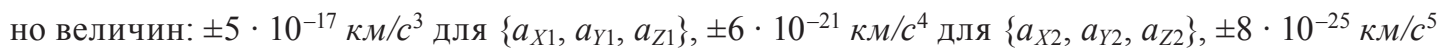
для $\left\{a_{X 3}, a_{Y 3}, a_{Z 3}\right\}, \pm 2 \cdot 10^{-29} \mathrm{kM} / \mathrm{c}^{6}$ для $\left\{a_{X 4}, a_{Y 4}, a_{Z 4}\right\}$, а критерием успешного решения задачи считали эквивалентные по точности решения, обеспечивающие расчет положения НКА с СКО от опорных эфемерид СВОЭВП на четырехчасовом интервале на уровне, втрое меньшем, чем уровень погрешности, заданный в Таблице К.1 [1] для долговременного алгоритма, т.е. не более $33,3 \mathrm{~cm}$.

По согласованной (в части скоростей и ускорений) ЭИ НКА и с использованием уточненных ПДМД проведен расчет положения НКА на интервале согласования $\left[t_{b} ; t_{b}+4\right.$ u $]$ и апосте- 
риорная оценка погрешности расчета положения НКА относительно опорных эфемерид SP3 СВОЭВП. Результаты оценок приведены ниже.

\section{Апостериорная оценка погрешности расчета положения НКА}

Предложенные методы определения вектора согласованных эфемерид и ПДМД были протестированы для НКА ОГ ГЛОНАСС для получасовых и четырехчасовых интервалов согласования за даты с 18.12.2016 по 21.12.2016.

На первом этапе были получены оценки скоростей и ускорений НКА на неперекрывающихся получасовых интервалах согласования $\left[t_{b}-0,25\right.$; $t_{b}+0,25$ u для опорных эпох $t_{b}$ на 15 -ю и 45-ю минуты часа.

Частные производные при оценке вектора $\{\dot{x}, \dot{y}, \dot{z}, \ddot{x}, \ddot{y}, \ddot{z}\}$ рассчитывали с шагом 5 с. Оценки отклонений положения НКА (евклидово расстояние) по согласованным эфемеридам НКА от опорных эфемерид SР3 СВОЭВП рассчитывали с шагом 1 с.

Среднеквадратические отклонения положений НКА, рассчитанных на интервале $\left[t_{b}-0,254 ; t_{b}+0,254\right]$ по согласованной (в части скоростей и ускорений) ЭИ НКА, от опорныХ эфемерид SР3 СВОЭВП изображены на рис. 1.

По результатам, приведенным на рис. 1, можно видеть, что погрешность расчета положения НКА (СКО) по согласованным в части скоростей и ускорений эфемеридам на 15 -минутном

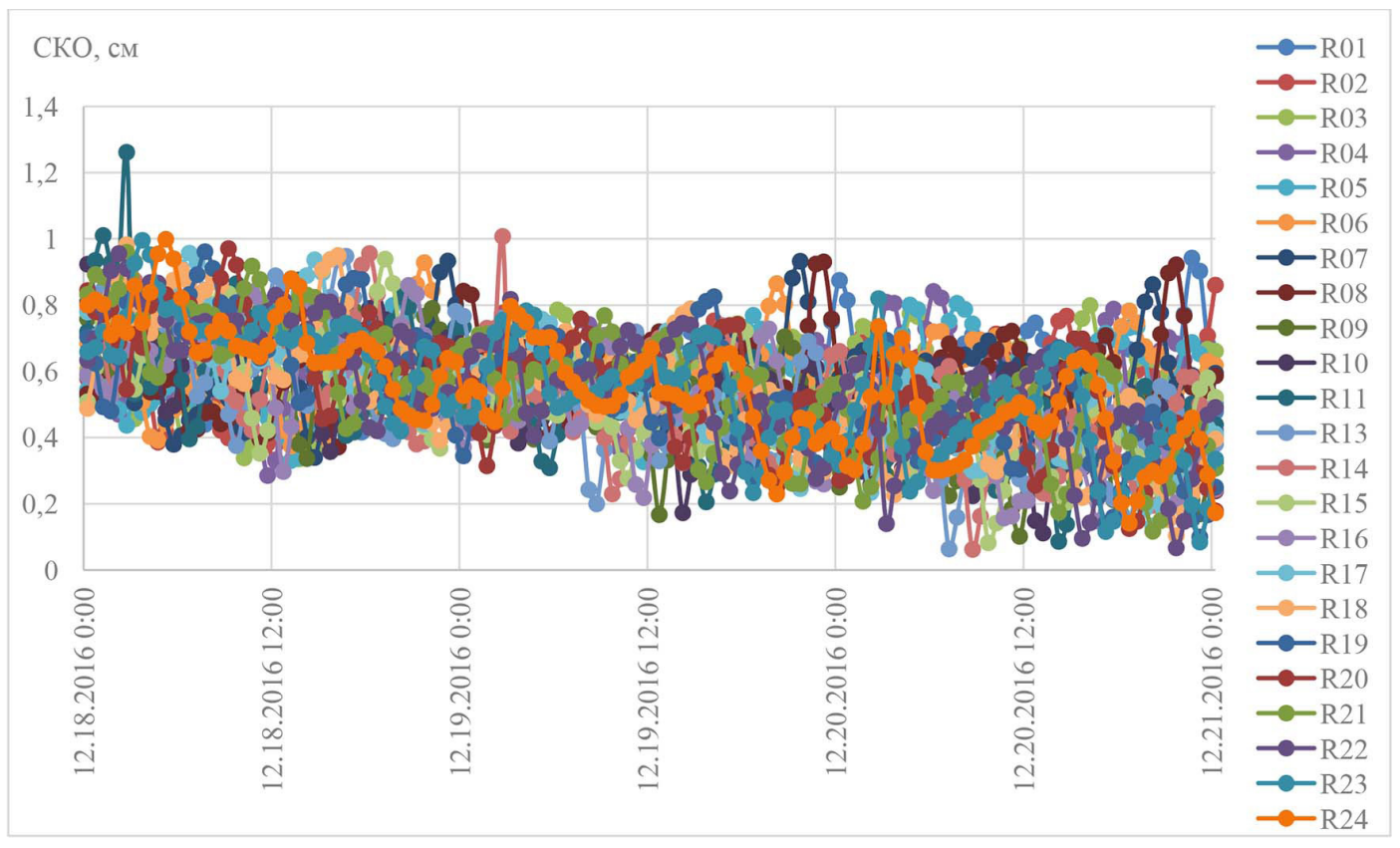

Рис. 1. Погрешности расчета положения НКА (СКО) по согласованной в части скоростей и ускорений ЭИ НКА ОГ ГЛОНАСС на интервалах согласования $\left[t_{b}-0,25 u ; t_{b}+0,25 u\right]$

Fig. 1. GLONASS SV (R01 ... R24) Position accuracy (standard deviations) after forecast in accordance with User Algorithm K.2.1 for Determination of SV Position [1]. Improved Ephemeris data sets $\left\{\dot{x}\left(t_{b}\right), \dot{y}\left(t_{b}\right), \dot{z}\left(t_{b}\right), \ddot{x}\left(t_{b}\right), \ddot{y}\left(t_{b}\right), \ddot{z}\left(t_{b}\right)\right\}$ are produced via a least squares curve fit of the SP3 SVOEVP ephemeris, fitting intervals are $\left.\left[t_{b}-0,25 u ; t_{b}+0,25 u\right]\right)$. The values of SV Position parameters $\left\{x\left(t_{b}\right), y\left(t_{b}\right), z\left(t_{b}\right)\right\}$ match with SP3 SVOEVP ephemeris 


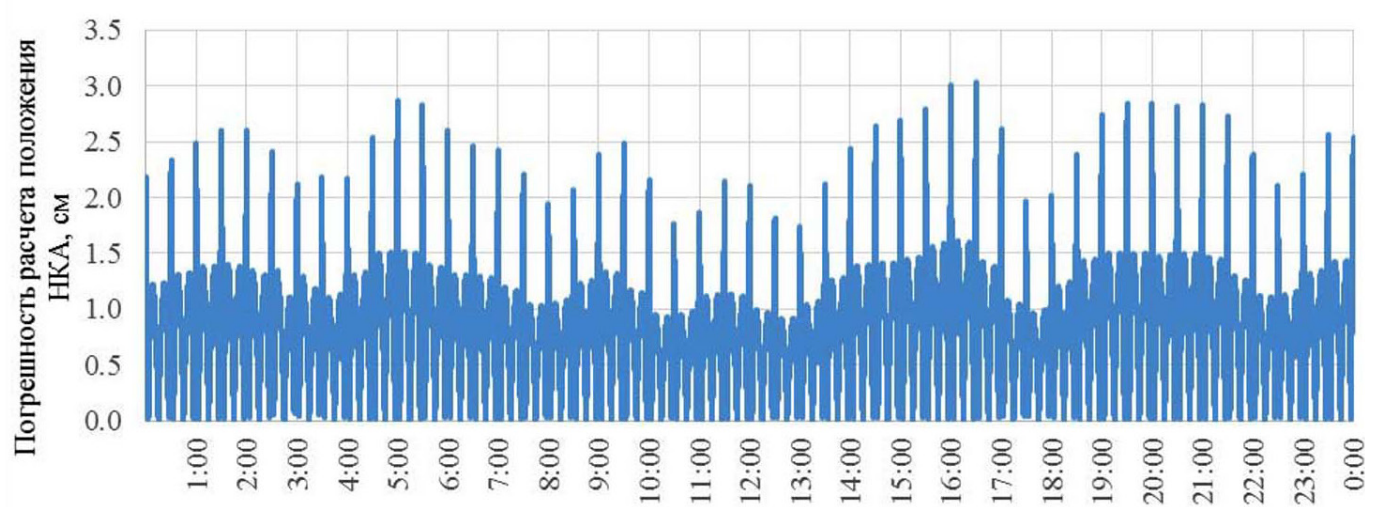

Рис. 2. Оценка погрешности расчета положения НКА (невязки) по согласованным эфемеридам для НКА ГЛОНАСС №730, системная точка 1, дата 18.12 .2016 , состав уточняемого вектора $\left\{\dot{x}\left(t_{b}\right), \dot{y}\left(t_{b}\right), \dot{z}\left(t_{b}\right), \ddot{x}\left(t_{b}\right), \ddot{y}\left(t_{b}\right), \ddot{z}\left(t_{b}\right)\right\}$

Fig. 2. Deviations of the calculated GLONASS SV R01 Positions from the exact SP3 SVOEVP ephemeris (December 18, 2016) after forecast on intervals $\left[t_{b} ; t_{b}+0,25 u\right]$ in accordance with User Algorithm K.2.1 for Determination of SV Position [1]. Ephemeris data sets $\mathrm{f}\left\{\dot{x}\left(t_{b}\right), \dot{y}\left(t_{b}\right), \dot{z}\left(t_{b}\right), \ddot{x}\left(t_{b}\right), \ddot{y}\left(t_{b}\right), \ddot{z}\left(t_{b}\right)\right\}$ are produced via a least squares curve fit of the SP3 SVOEVP ephemeris, the values of SV Position parameters $\left\{x\left(t_{b}\right), y\left(t_{b}\right), z\left(t_{b}\right)\right\}$ match with SP3 SVOEVP ephemeris

интервале от опорной эпохи $t_{b}$ не превысила 1 см, за исключением двух реализаций из 3480, которые составили 1,26 и 1,01 см. Следовательно, согласованная эфемеридная информация НКА ГЛОНАСС позволит обеспечить требование [1] по точности расчета положения НКА с погрешностью не более 5 см на всем получасовом интервале согласования.

На рис. 2 приведены результаты оценки погрешности отклонения положения НКА (невязки) по согласованным эфемеридам для НКА ГЛОНАСС №730, системная точка 1, дата 18.12.2016, в качестве эталонных эфемерид использованы опорные эфемериды SР3 СВОЭВП. Интервалы расчета положения НКА для каждой опорной эпохи $t_{b}:\left[t_{b}-0,254 ; t_{b}+0,25 u\right]$, состав уточняемого вектора $\left\{\dot{x}\left(t_{b}\right), \dot{y}\left(t_{b}\right), \dot{z}\left(t_{b}\right), \ddot{x}\left(t_{b}\right), \ddot{y}\left(t_{b}\right), \ddot{z}\left(t_{b}\right)\right\}$. Погрешность расчета положения НКА по согласованным эфемеридам (в части скоростей и ускорений) составила не более $3 \mathrm{~cm}$ на 15 -минутном интервале от момента $t_{b}$. Сопоставимые по точности расчета положения НКА результаты получены по другим НКА ГЛОНАСС.

Тестовые расчеты показали, что если на опорные эпохи $t_{b}$ используются окончательные опорные эфемериды SР3 СВОЭВП и скорости, полученные интерполяцией эфемерид SP3 CВОЭВП на соответствующие опорные эпохи $t_{b}$, а в состав уточняемого вектора входят только ускорения НКА $\ddot{x}\left(t_{b}\right), \ddot{y}\left(t_{b}\right), \ddot{z}\left(t_{b}\right)$, погрешность расчета положения НКА по алгоритму потребителя [1] на 15 минутном интервале в большей части реализаций превышает 5 см, т.е. требование [1] по точности расчета положения НКА по согласованной ЭИ не удовлетворяется.

На рис. 3 отражены результаты оценки отклонения расчетного положения НКА ГЛОНАСС №730 по согласованной (только в части ускорений) ЭИ, системная точка 1, дата 18.12.2016, в качестве эталонных использованы опорные эфемериды СВОЭВП и компоненты скорости, рассчитанные при помощи интерполяционного многочлена Лагранжа 12-го порядка. Интервалы расчета положения НКА для каждой опорной эпохи $t_{b}$ : $\left[t_{b}-0,25 y ; t_{b}+0,25 u\right]$, состав уточняемого вектора $\left\{\ddot{x}\left(t_{b}\right), \ddot{y}\left(t_{b}\right), \ddot{z}\left(t_{b}\right)\right\}$. Погрешность расчета положения НКА только для двух реализа- 


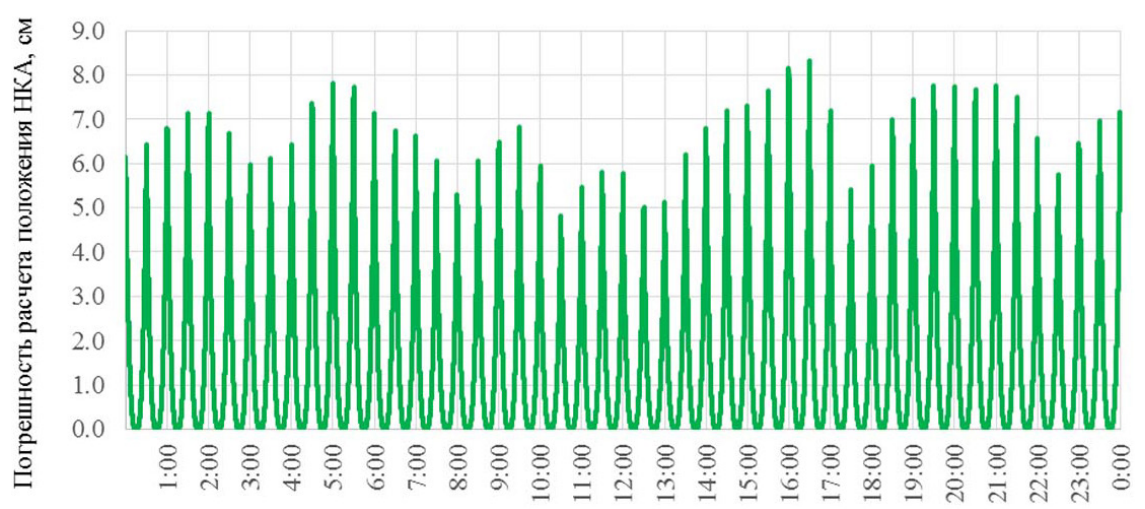

Рис. 3. Оценка погрешности расчета положения НКА по согласованной (только в части ускорений) ЭИ для НКА ГЛОНАСС №730, системная точка 1 , дата 18.12 .2016 , состав уточняемого вектора $\left\{\ddot{x}\left(t_{b}\right), \ddot{y}\left(t_{b}\right), \ddot{z}\left(t_{b}\right)\right\}$ (координаты и скорости НКА на моменты $t_{b}$ соответствуют опорным эфемеридам SP3)

Fig. 3. Deviations of the calculated GLONASS SV R01 Positions from the exact SP3 SVOEVP ephemeris (December $18,2016)$ after forecast on intervals $\left[t_{b} ; t_{b}+0,25 u\right]$ in accordance with User Algorithm K.2.1 for Determination of SV Position [1]. Ephemeris data sets $\left\{\ddot{x}\left(t_{b}\right), \ddot{y}\left(t_{b}\right), \ddot{z}\left(t_{b}\right)\right\}$ are produced via a least squares curve fit of the SP3 SVOEVP ephemeris, the values of SV position \& velocity parameters $\left\{x\left(t_{b}\right), y\left(t_{b}\right), z\left(t_{b}\right), \dot{x}\left(t_{b}\right), \dot{y}\left(t_{b}\right), \dot{z}\left(t_{b}\right)\right\}$ match with SP3 SVOEVP ephemeris

ций уточнения из 48 не превысила заданное в [1] требование по точности расчета положения НКА по согласованной ЭИ, в большей части реализаций погрешность расчета положения НКА на интервалах интегрирования до 15 мин от опорной эпохи $t_{b}$ составила до $6 \ldots 8$ см.

Таким образом, с точки зрения обеспечения требования [1] по точности расчета положения НКА ГЛОНАСС вариант формирования согласованной ЭИ на интервале $\left[t_{b}-0,254 ; t_{b}+0,25 u\right]$, когда в состав уточняемого вектора включены как компоненты скорости, так и компоненты ускорения НКА, а в качестве координат НКА используются опорные эфемериды SP3 на момент $t_{b}$, предпочтительнее.

Исключение из состава уточняемого вектора компонент скоростей и замена уточненных компонент скорости НКА $\left\{\dot{x}\left(t_{b}\right), \dot{y}\left(t_{b}\right), \dot{z}\left(t_{b}\right)\right\}$ на компоненты скорости, полученные интерполированием опорных эфемерид SP3, приводит к увеличению погрешности расчета положения НКА на интервале $\left[t_{b}-0,25\right.$ ч; $t_{b}+0,25$ ч] до $2 \ldots 3$ раз.

На 2-м этапе были получены оценки погрешности расчета положения НКА на основе согласованной ЭИ и уточненных ПДМД для опорных эпох $t_{b}$ на 15-ю и 45-ю минуты часа на интервалах согласования $\left[t_{b} ; t_{b}+4\right.$ u]. Задача решалась итерационным методом Ньютона с использованием устойчивых регуляризирующих алгоритмов по А.Н. Тихонову [7, 8], описанных выше. При оценке вектора $\left\{a_{X 1}, a_{X 2}, a_{X 3}, a_{X 4}, a_{Y 1}, a_{Y 2}, a_{Y 3}, a_{Y 4}, a_{Z 1}, a_{Z 2}, a_{Z 3}, a_{Z 4}\right\}$ частные производные на интервале согласования 4 ч рассчитывали с шагом $60 \mathrm{c}$, при интегрировании уравнений движения НКА ГЛОНАСС использовали долговременный алгоритм [1].

Для оценки устойчивости решения задачи были проведены расчеты с различными начальными априорными значениями вектора $\left\{a_{X 1}, a_{X 2}, a_{X 3}, a_{X 4}, a_{Y 1}, a_{Y 2}, a_{Y 3}, a_{Y 4}, a_{Z 1}, a_{Z 2}\right.$, $\left.a_{Z 3}, a_{Z 4}\right\}$ - с нулевыми значениями и с возмущенными, ненулевыми значениями на уровне $\sim 5 \cdot 10^{-14} \kappa M / c^{3}$ для $\left|a_{X 1}{ }^{(0)}\right|,\left|a_{Y 1}{ }^{(0)}\right|,\left|a_{Z 1}{ }^{(0)}\right|, \sim 5 \cdot 10^{-18} \kappa M / c^{4}$ для $\left|a_{X 2}{ }^{(0)}\right|,\left|a_{Y 2}{ }^{(0)}\right|,\left|a_{Z 2}{ }^{(0)}\right|, \sim 5 \cdot 10^{-22} \kappa M / c^{5}$ для $\left|a_{X 3}{ }^{(0)}\right|,\left|a_{Y 3}{ }^{(0)}\right|,\left|a_{Z 3}{ }^{(0)}\right|, \sim 5 \cdot 10^{-27} \kappa M / c^{6}$ для $\left|a_{X 4}{ }^{(0)}\right|,\left|a_{Y 4}{ }^{(0)}\right|,\left|a_{Z 4}{ }^{(0)}\right|$.

$$
-797-
$$


Расчет отклонений положения НКА, рассчитанной по согласованной ЭИ и ПДМД НКА, от положения НКА, рассчитанного по опорным эфемеридам SP3 СВОЭВП по интерполяционным полиномам Лагранжа, производили с шагом 1 c.

Для оценки эффективности использования метода регуляризации был также реализован вариант определения вектора $\left\{a_{X 1}, a_{X 2}, a_{X 3}, a_{X 4}, a_{Y 1}, a_{Y 2}, a_{Y 3}, a_{Y 4}, a_{Z 1}, a_{Z 2}, a_{Z 3}, a_{Z 4}\right\}$ с использованием классического МНК. При использовании указанного метода при оценке вектора $\left\{a_{X 1}, a_{X 2}\right.$, $\left.a_{X 3}, a_{X 4}, a_{Y 1}, a_{Y 2}, a_{Y 3}, a_{Y 4}, a_{Z 1}, a_{Z 2}, a_{Z 3}, a_{Z 4}\right\}$ для части реализаций возможно получить решения системы уравнений, сопоставимые с теми, которые были получены с применением регуляризирующих алгоритмов - погрешность расчета положения НКА на интервале согласования 4 ч может не превышать $0,10 \ldots 0,25$ м.

Однако процесс решения системы условных уравнений с применением классического МНК, а не регуляризирующих алгоритмов является неустойчивым. Конечные результаты оценки вектора $\left\{a_{X 1}, a_{X 2}, a_{X 3}, a_{X 4}, a_{Y 1}, a_{Y 2}, a_{Y 3}, a_{Y 4}, a_{Z 1}, a_{Z 2}, a_{Z 3}, a_{Z 4}\right\}$ с использованием классического МНК зависят от уровня возмущения начального априорного значения уточняемого вектора, критерий сходимости для прекращения итерационного уточнения вектора ПДМД для части реализаций может быть не выполнен. Тестовые расчеты показали, что погрешность расчета положения НКА на интервале согласования 4 ч относительно опорных эфемерид SP3 CВОЭВП может достигать 25 м и более, если применены ПДМД, уточненные с использованием классического МНК.

Таким образом, в случае применения классического МНК не все реализации уточнения вектора $\left\{a_{X 1}, a_{X 2}, a_{X 3}, a_{X 4}, a_{Y 1}, a_{Y 2}, a_{Y 3}, a_{Y 4}, a_{Z 1}, a_{Z 2}, a_{Z 3}, a_{Z 4}\right\}$ обеспечивают эквивалентные по точности расчеты положения НКА на интервале согласования, а для ряда реализаций уточнения указанного вектора не выполняется требование [1] по точности.

В качестве примера на рис. 4 приведены результаты оценки погрешности расчета положения НКА для варианта применения классического МНК без регуляризации для уточнения вектора $\left\{a_{X 1}, a_{X 2}, a_{X 3}, a_{X 4}, a_{Y 1}, a_{Y 2}, a_{Y 3}, a_{Y 4}, a_{Z 1}, a_{Z 2}, a_{Z 3}, a_{Z 4}\right\}$ с нулевыми и возмущенными значениями, задаваемыми в качестве начального априорного значения вектора.

Апостериорные оценки погрешности расчета положения НКА показали, что при применении регуляризирующих алгоритмов по А.Н. Тихонову для всех реализаций решения задачи обеспечиваются эквивалентные точности вне зависимости от того, заданы ли начальные априорные значения вектора $\left\{a_{X 1}, a_{X 2}, a_{X 3}, a_{X 4}, a_{Y 1}, a_{Y 2}, a_{Y 3}, a_{Y 4}, a_{Z 1}, a_{Z 2}, a_{Z 3}, a_{Z 4}\right\}$ до начала уточнения нулевыми или возмущенными.

На рис. 5, 6 приведены результаты оценки точности расчета положения НКА по согласованной (в части скоростей и ускорений) ЭИ и ПДМД - соответственно среднеквадратические отклонения (СКО) и абсолютные отклонения положения НКА ОГ ГЛОНАСС от опорных эфемерид SР3 СВОЭВП (невязки) при расчете положения НКА на интервалах согласования $\left[t_{b} ; t_{b}+4 u\right]$ для варианта, в котором для определения вектора $\left\{a_{X 1}, a_{X 2}, a_{X 3}, a_{X 4}, a_{Y 1}, a_{Y 2}, a_{Y 3}, a_{Y 4}\right.$, $\left.a_{Z 1}, a_{Z 2}, a_{Z 3}, a_{Z 4}\right\}$ использованы устойчивые регуляризирующие алгоритмы по А.Н. Тихонову.

Погрешность расчета положения НКА (СКО) в интервалах до 4 ч от опорной эпохи $t_{b}$ по алгоритмам ИКД по согласованной эфемеридной информации в части параметров $\left\{a_{X 1}, a_{X 2}\right.$, $\left.a_{X 3}, a_{X 4}, a_{Y 1}, a_{Y 2}, a_{Y 3}, a_{Y 4}, a_{Z 1}, a_{Z 2}, a_{Z 3}, a_{Z 4}\right\}$ для рассматриваемого интервала моделирования не превысила 6 см, при этом для более $95 \%$ реализаций обеспечивался расчет положения НКА

$$
-798-
$$




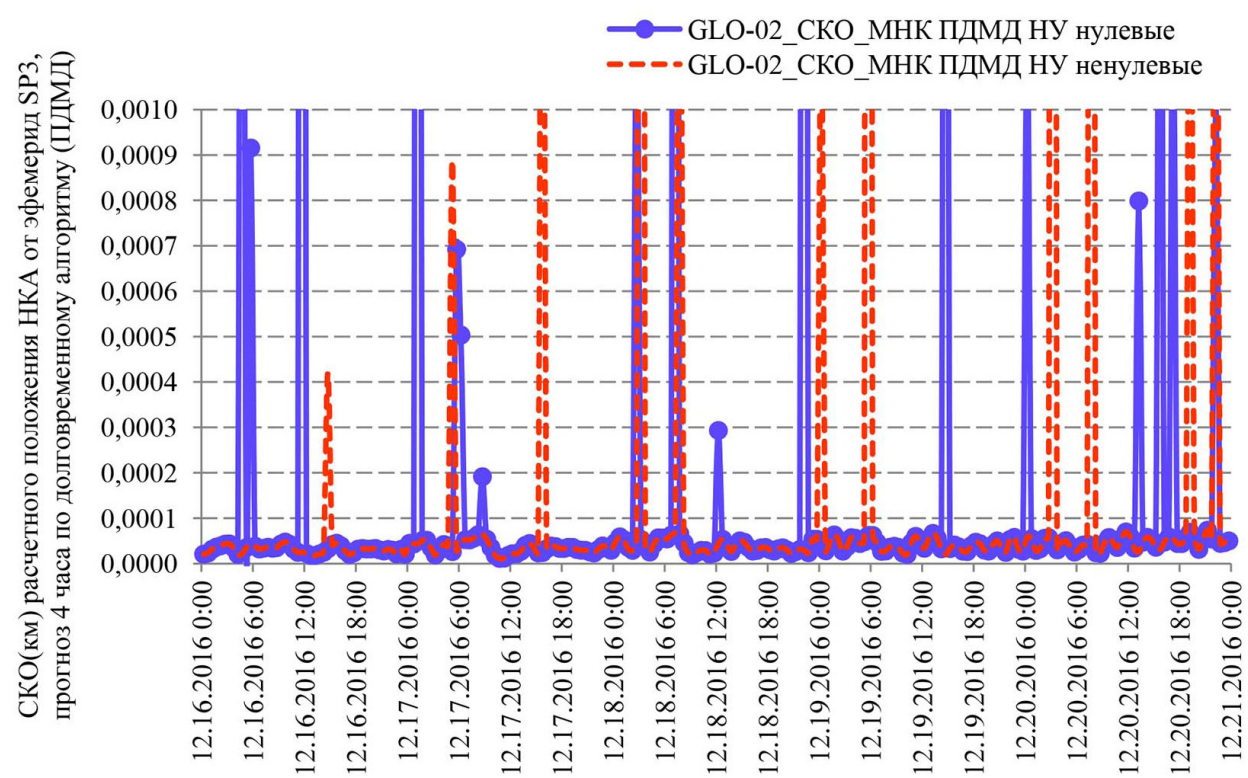

Рис. 4. Оценка погрешности расчета положения НКА (СКО) на интервалах $\left[t_{b} ; t_{b}+4\right.$ ч] по согласованной ЭИ для НКА ГЛОНАСС №747, системная точка 2, даты 16.12.2016 - 20.12.2016, состав уточняемого вектора $\left\{a_{X 1}, a_{X 2}, a_{X 3}, a_{X 4}, a_{Y 1}, a_{Y 2}, a_{Y 3}, a_{Y 4}, a_{Z 1}, a_{Z 2}, a_{Z 3}, a_{Z 4}\right\}$, применен классический МНК, без регуляризации

Fig. 4. GLONASS SV R02 Position accuracy (standard deviations) after forecast on intervals $\left[t_{b} ; t_{b}+4 u\right]$ in accordance with User Algorithm K.3.1 for Determination of SV Position [1], December 16 - December 18, 2016. The data sets $\left\{\dot{x}\left(t_{b}\right), \dot{y}\left(t_{b}\right), \dot{z}\left(t_{b}\right), \ddot{x}\left(t_{b}\right), \ddot{y}\left(t_{b}\right), \ddot{z}\left(t_{b}\right)\right\}$ and the Long-term model parameter sets $\left\{a_{X 1}, a_{X 2}, a_{X 3}, a_{X 4}, a_{Y 1}\right.$, $\left.a_{Y 2}, a_{Y 3}, a_{Y 4}, a_{Z 1}, a_{Z 2}, a_{Z 3}, a_{Z 4}\right\}$ for L1OC, L3OC navigation message are produced only via a least squares curve fit of the SP3 SVOEVP ephemeris. Fitting intervals are $\left[t_{b}-0,25 u ; t_{b}+0,25 u\right]$ and $\left[t_{b} ; t_{b}+4 u\right]$ respectively for the SV position \& velocity parameter sets and the Long-term model parameter sets

со среднеквадратическим отклонением от опорных эфемерид SP3 СВОЭВП, не превышающим $3 . .5 \mathrm{~cm}$.

Все реализации расчета положения НКА по согласованной в части скоростей и ускорений ЭИ и ПДМД $\left\{a_{X 1}, a_{X 2}, a_{X 3}, a_{X 4}, a_{Y 1}, a_{Y 2}, a_{Y 3}, a_{Y 4}, a_{Z 1}, a_{Z 2}, a_{Z 3}, a_{Z 4}\right\}$, рассчитанных с использованием регуляризирующих алгоритмов по А.Н. Тихонову, эквивалентны по точности и обеспечили расчет положения НКА на интервалах $\left[t_{b} ; t_{b}+4 u\right]$ с погрешностью, не превышающей 0,2 м.

Исходя из вышеизложенного можно сделать вывод, что верхняя граница требования [1] по погрешности расчета положения НКА по долговременному алгоритму не более 1 м на интервалах расчета положения НКА до +4 ч от опорной эпохи $t_{b}$ обеспечивается с запасом.

\section{Заключение}

В данной работе представлены способы оценки вектора $\left\{\dot{x}\left(t_{b}\right), \dot{y}\left(t_{b}\right), \dot{z}\left(t_{b}\right), \ddot{x}\left(t_{b}\right), \ddot{y}\left(t_{b}\right), \ddot{z}\left(t_{b}\right)\right\}$ НКА ГЛОНАСС и вектора параметров долговременной модели движения НКА ГЛОНАСС $\left\{a_{X 1}\right.$, $\left.a_{X 2}, a_{X 3}, a_{X 4}, a_{Y 1}, a_{Y 2}, a_{Y 3}, a_{Y 4}, a_{Z 1}, a_{Z 2}, a_{Z 3}, a_{Z 4}\right\}$, обеспечивающих расчет параметров движения НКА ГЛОНАСС по алгоритмам [1] с высокой точностью на интервалах согласования соответственно до \pm 15 мин и до +4 ч от опорной эпохи $t_{b}$.

Показано, что параметры движения НКА ГЛОНАСС в составе опорных эфемеридНКАГЛОНАСС SP3 СВОЭВП $x\left(t_{b}\right), y\left(t_{b}\right), z\left(t_{b}\right)$, скоростей и ускорений НКА $\left\{\dot{x}\left(t_{b}\right), \dot{y}\left(t_{b}\right), \dot{z}\left(t_{b}\right), \ddot{x}\left(t_{b}\right), \ddot{y}\left(t_{b}\right), \ddot{z}\left(t_{b}\right)\right\}$, 


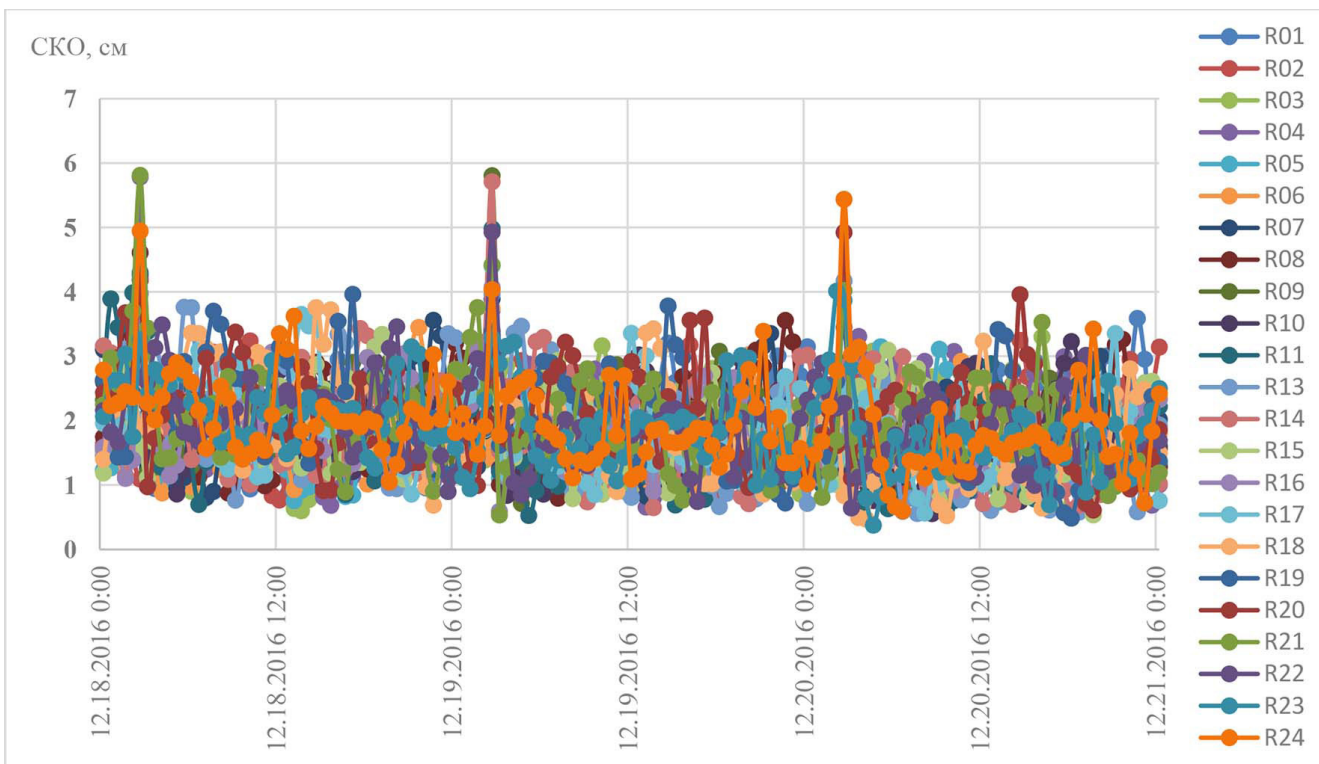

Рис. 5. Оценка погрешности расчета положения НКА (СКО) на интервалах $\left[t_{b} ; t_{b}+4\right.$ u по согласованной ЭИ и ПДМД для НКА ОГ ГЛОНАСС, даты 18.12.2016 - 20.12.2016. ЭИ НКА ОГ ГЛОНАСС согласована в части скоростей и ускорений. Состав уточняемого вектора ПДМД $\left\{a_{X 1}, a_{X 2}, a_{X 3}, a_{X 4}, a_{Y 1}, a_{Y 2}, a_{Y 3}, a_{Y 4}, a_{Z 1}\right.$, $\left.a_{Z 2}, a_{Z 3}, a_{Z 4}\right\}$, применен метод регуляризации по А.Н. Тихонову

Fig. 5. GLONASS SVs (R01 ... R24) Position accuracy (standard deviations) after forecast on intervals $\left[t_{b} ; t_{b}+4 u\right]$ in accordance with User Algorithm K.3.1 for Determination of SV Position [1], December 16 - December 18, 2016. The Long-term model parameter sets $\left\{a_{X 1}, a_{X 2}, a_{X 3}, a_{X 4}, a_{Y 1}, a_{Y 2}, a_{Y 3}, a_{Y 4}, a_{Z 1}, a_{Z 2}, a_{Z 3}, a_{Z 4}\right\}$ for L1OC, L3OC navigation message are produced via A. N. Tikhonov regularizing method curve fit of the SP3 SVOEVP ephemeris, fitting intervals are $\left[t_{b} ; t_{b}+4 u\right]$

уточненных на интервале $\left[t_{b}-0,25\right.$ ч; $t_{b}+0,25$ ч], и ПДМД НКА $\left\{a_{X 1}, a_{X 2}, a_{X 3}, a_{X 4}, a_{Y 1}, a_{Y 2}, a_{Y 3}\right.$, $\left.a_{Y 4}, a_{Z 1}, a_{Z 2}, a_{Z 3}, a_{Z 4}\right\}$, уточненных на интервалах $\left[t_{b} ; t_{b}+4\right.$ u с применением регуляризирующего алгоритма А.Н. Тихонова, позволяют обеспечить расчет положения НКА по алгоритмам [1] с погрешностью не более единиц дециметров на интервалах согласования до 4 ч - в тестовых расчетах погрешность расчета положения НКА ОГ ГЛОНАСС не превысила 0,2 м.

Оценена эффективность применения регуляризирующего алгоритма А.Н. Тихонова по сравнению с результатами, полученными при использовании классического МНК.

Следует отметить, что решения для вектора $\left\{a_{X 1}, a_{X 2}, a_{X 3}, a_{X 4}, a_{Y 1}, a_{Y 2}, a_{Y 3}, a_{Y 4}, a_{Z 1}, a_{Z 2}, a_{Z 3}\right.$, $\left.a_{Z 4}\right\}$, полученные с использованием классического МНК, неустойчивы, результаты оценки вышеуказанного вектора существенно зависят от начальных априорных значений уточняемых параметров и не гарантируют точность расчета параметров движения НКА, эквивалентных по точности решениям по регуляризирующему алгоритму. Погрешности расчета положения НКА с ПДМД, уточненными по классическому МНК, относительно эталонных эфемерид SP3 СВОЭВП (евклидово расстояние) на интервалах до +4 ч от опорной эпохи $t_{b}$ для некоторых реализаций уточнения ПДМД могут достигать 20 м ... 25 м.

Представленный выше метод определения ПДМД НКА ГЛОНАСС $\left\{a_{X 1}, a_{X 2}, a_{X 3}, a_{X 4}, a_{Y 1}\right.$, $\left.a_{Y 2}, a_{Y 3}, a_{Y 4}, a_{Z 1}, a_{Z 2}, a_{Z 3}, a_{Z 4}\right\}$ с использованием регуляризирующего алгоритма А.Н. Тихонова может быть применен в программном обеспечении генерации навигационной цифровой ин- 


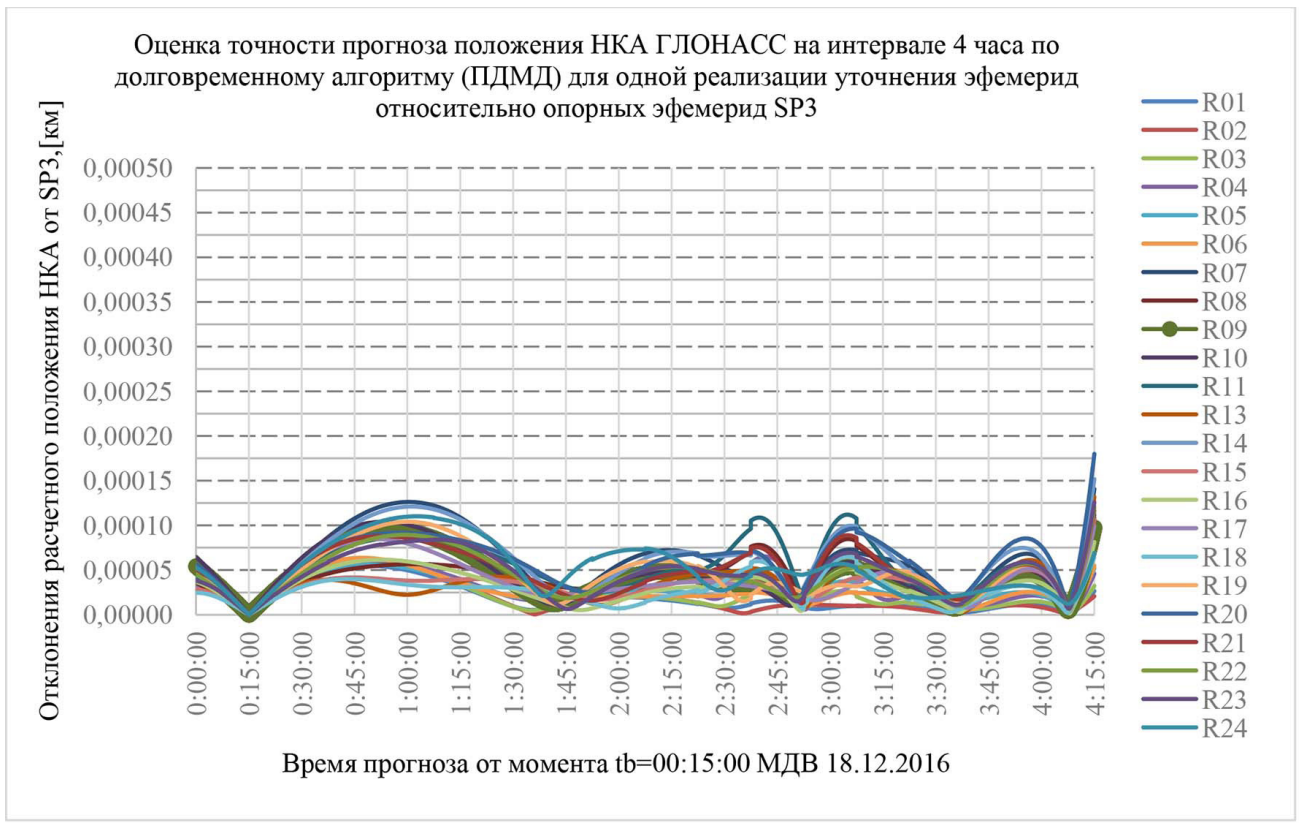

Рис. 6. Оценка погрешности расчета положения НКА (невязки) на интервале $\left[t_{b} ; t_{b}+4\right.$ u по согласованной ЭИ и ПДМД для НКА ОГ ГЛОНАСС, дата 18.12.2016, $t_{b}$ 00:15:00 МДВ. ЭИ НКА ОГ ГЛОНАСС согласована в части скоростей и ускорений. Состав уточняемого вектора ПДМД $\left\{a_{X 1}, a_{X 2}, a_{X 3}, a_{X 4}, a_{Y 1}, a_{Y 2}, a_{Y 3}, a_{Y 4}\right.$, $\left.a_{Z 1}, a_{Z 2}, a_{Z 3}, a_{Z 4}\right\}$, применен метод регуляризации по А.Н. Тихонову

Fig. 6. Deviations of the calculated GLONASS SV (R01 ... R24) Positions from the exact SP3 SVOEVP ephemeris (December 18, 2016) after forecast on intervals $\left[t_{b} ; t_{b}+4 u\right]$ in accordance with User Algorithm K.3.1 for Determination of SV Position [1]. Ephemeris data sets for L1OC, L3OC navigation message are used: the values of SV Position parameters $\left\{x\left(t_{b}\right), y\left(t_{b}\right), z\left(t_{b}\right)\right\}$ match with SP3 SVOEVP ephemeris; improved data sets $\left\{\dot{x}\left(t_{b}\right), \dot{y}\left(t_{b}\right), \dot{z}\left(t_{b}\right), \ddot{x}\left(t_{b}\right), \ddot{y}\left(t_{b}\right), \ddot{z}\left(t_{b}\right)\right\}$ are produced via a least squares curve fit of the SP3 SVOEVP ephemeris; the Long-term model parameter sets $\left\{a_{X 1}, a_{X 2}, a_{X 3}, a_{X 4}, a_{Y 1}, a_{Y 2}, a_{Y 3}, a_{Y 4}, a_{Z 1}, a_{Z 2}, a_{Z 3}, a_{Z 4}\right\}$ are produced via A.N. Tikhonov regularizing method curve fit of the SP3 SVOEVP ephemeris

формации L1OC, L3OC ГЛOHACC, предназначенной для тестирования навигационных приемников с целью оценки погрешности алгоритмов определения местоположения.

Указанный метод устойчивого определения ПДМД может быть также применен в программном обеспечении НКА «Глонасс-К2» при формировании навигационной цифровой информации L1OC, L3OC ГЛОНАCC на борту НКА.

\section{Список литературы / References}

[1] ГЛОНАСС. Интерфейсный контрольный документ. Общее описание системы с кодовым разделением сигналов. Редакция 1.0. Сайт ОАО «Российские космические системы». http://russianspacesystems.ru/wp-content/uploads/2016/08/IKD.-Obshh.-opis.-Red.-1.0-2016.pdf [GLONASS. Interface control document. General Description of Code Division Multiple Access Signal System. Edition 1.0. The website: http://russianspacesystems.ru/wp-content/uploads/2016/08/ ICD-GLONASS-CDMA-General.-Edition-1.0-2016.pdf], дата обращения: 30.04.2019.

[2] Сайт СВОЭВП. Access: http://glonass-svoevp.ru/index.php?option=com_content\&view=art icle\&id=55\&Itemid=259\&lang=ru [The website. Access: http://glonass-svoevp.ru], дата обращения: 15.08.2018. 
[3] Бахвалов Н.С., Жидков Н.П., Кобельков Г.М. Численные методы. М.: БИНОМ. Лаборатория знаний, 2008. 636 с [Bakhvalov N.S., Zhidkov N.P., Kobelkov G.M. Numerical methods. Moscow, BINOM. Knowledge laboratory, 2008. 636 p. (in Russian)].

[4] Форсайт Дж.Е., Малькольм М.А., Моулер К.Б. Машинные методы математических вычислений. М.: Мир, 1980. 280 с. [Forsythe G.E., Malcolm M.A., Moler C.B. Computer methods for mathematical computations. Moscow, Mir, 1980. 280 p. (in Russian)].

[5] Лоусон Ч., Хенсон Р. Численное решение задач методом наименьших квадратов. М.: Наука, 1986. 230 c. [Lawson C.L., Hanson R.J. Solving Least Squares Problems. Moscow, Nauka, 1986, 230 p. (in Russian)].

[6] Берсенев С.М. О пересчете факторизации Холецкого. Журн. вычисл. матем. и матем. физ., 1979, 19, (5), 1318-1319. [Bersenev S.M. Algorithmic variant for Cholesky factorization. USSR Computational Mathematics and Mathematical Physics, 1979, 19(5), 1318-1319 (in Russian)].

[7] Тихонов А.Н. Об устойчивости алгоритмов для решения вырожденных систем линейных алгебраических уравнений. Журн. вычисл. матем. и матем. физ., 1965, 5(4), 718-722. [Tikhonov A.N. The stability of algorithms for the solution of degenerate systems of linear algebraic equations. USSR Computational Mathematics and Mathematical Physics, 1965, 5(4), 181-188 (in Russian)].

[8] Тихонов А.Н., Гончарский А.В., Степанов В.В., Ягола А.Г. Численные методы решения некорректных задач. М.: Наука, 1990. 229 с. [Tikhonov A.N., Goncharsky A.V., Stepanov V.V., Yagola A.G. Numerical methods for solving ill-posed problems. Moscow, Nauka, 1990, 229 c. (in Russian)]. 